\title{
Characterization of neocortical angioarchitecture across layers and areas in the human brain
}

Sven Hildebrand $^{1}$, Philipp Bethge ${ }^{2,3}$, Fabian F. Voigt ${ }^{2,3}$, Fritjof Helmchen ${ }^{2,3}$, Alard Roebroeck ${ }^{1}$

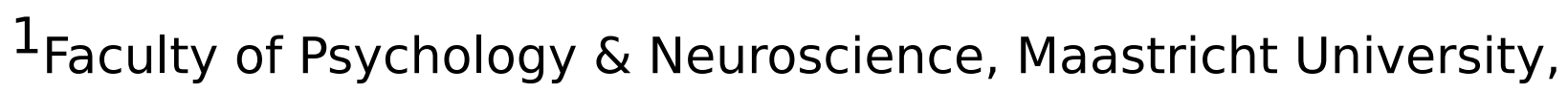
Maastricht, Netherlands. ${ }^{2}$ Brain Research Institute, University of Zurich, Zurich, Switzerland. ${ }^{3}$ Neuroscience Center Zurich, University of Zurich, ETH Zurich, Zurich, Switzerland

\section{Abstract Text}

The vasculature in human neocortex forms an intricate, dense network to provide sufficient supply of nutrients and oxygen to neuronal and glial cells. It is therefore to be expected, that cortical regions or laminae, which are particularly cell-rich, demand a higher microvessel density in their direct vicinity. Indeed, it has been shown that the general horizontal (tangential) architecture of intracortical blood vessels is organized in four distinct layers, which are at least partially related to the corresponding cytoarchitectural layers of the cortex. These intra- and interareal changes of vascular density are not yet well quantified in the human brain and could be of high interest for other fields of research. For instance laminar functional MRI (fMRI) at ultra-high fields, which refers to $7 \mathrm{~T}$ and above, is now enabling researchers to investigate feedback and feedforward signals within areas of interest, based on the depth of the fMRI signal within the cortical ribbon. However, the signal in fMRI is only indirectly related to actual neuronal activity and heavily dependent of the supplying vasculature. Hence, a more detailed understanding of factors such as the proportion of blood volume within large vessels versus capillaries, their distribution over the cortical sheet, and differences in this distribution over areas would be highly valuable. These factors could then be used as an anatomical foundation for better modeling of those laminar compartments. 
Furthermore, a precise definition of human cortical microvasculature could provide insights into vascular disease and microbleeds in the ageing brain.

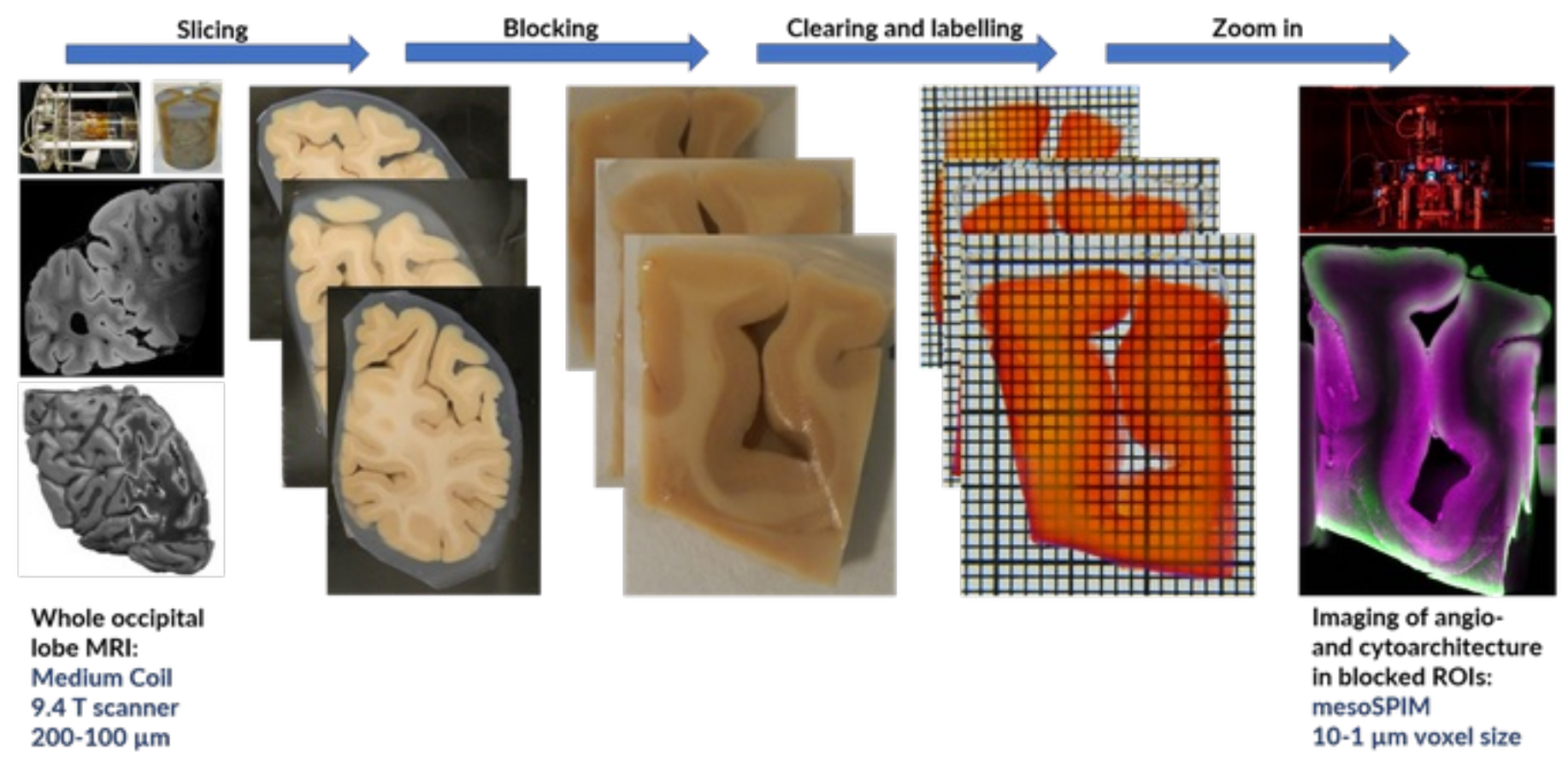

Figure 1: Overview of the imaging and tissue-processing pipeline.

Here we present multi-scale data of human occipital lobe tissue imaged both with ultra-high field MRI as well as light sheet fluorescence microscopy. First, the complete occipital lobes were scanned in a custom made RF-coil in a 9.4 T scanner, at a resolution of 100-200 $\mu \mathrm{m}$. To allow for volumetric fluorescent imaging at the meso- and microscale, we developed a method for homogenously labelling angio- and cytoarchitecture based on the recently published MASH clearing protocol ${ }^{1}$. Blocked regions of interest around the calcarine sulcus were then imaged in a mesoSPIM ${ }^{2}$ set-up at isotropic resolutions of 10-1 $\mu \mathrm{m}$ voxel size (see figure 1 ). This comprehensive multi-modal and multi-scale pipeline will enable a detailed comparison of the neocortical vasculature in V1 and V2 (figure 2) as well as other areas in the future. 


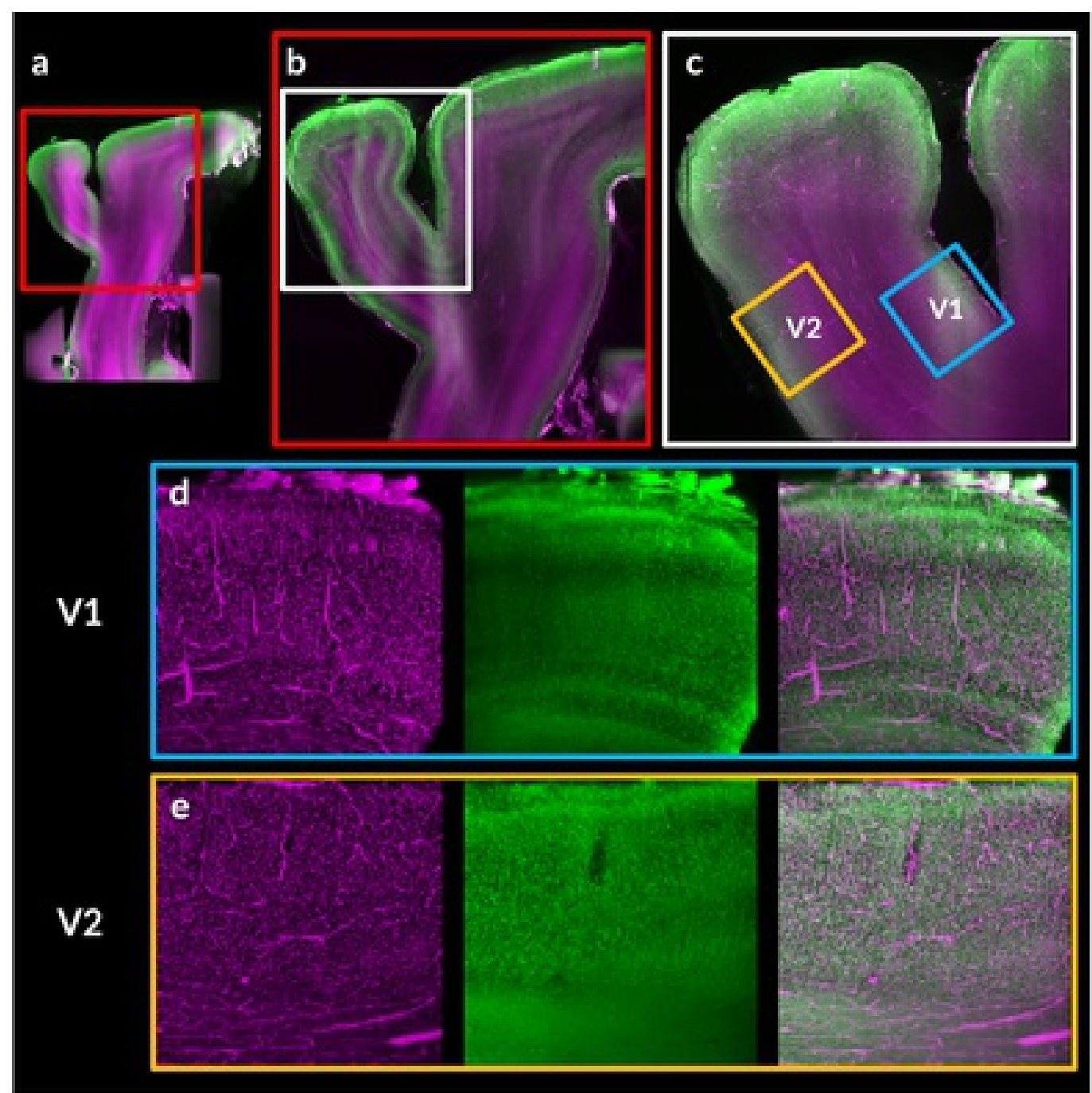

Figure 2: Representative multi-scale data set acquired on the mesoSPIM set-up. a) maximum intensity projection (MIP) over $50 \mu \mathrm{m}$ with the highest field of view (FOV) and a voxel size of approx. $10 \mu \mathrm{m}$. b) Layers start become discernible and $\mathrm{V} 1 / \mathrm{V} 2$ border is clearly visible at approx. $6 \mu \mathrm{m}$ voxel size (MIP over $30 \mu \mathrm{m}$ ). At the highest resolutions of $2 \mu \mathrm{m}$ (c) and $1 \mu \mathrm{m}$ (d and $\mathbf{e})$, the different angio- and cytoarchitectural layers and the differences in vascularization between $\mathrm{V} 1$ and $\mathrm{V} 2$ become clearly visible. 